\title{
Differential and developmental stage specific abundance of Zmdreb2a mRNA transcripts under drought stress and root development in Zea mays (L.)
}

\author{
Rakesh Sharma ${ }^{1}$, Manan Azad ${ }^{1}$, Vandana Nunia ${ }^{1}$, S.L. Kothari ${ }^{2}$, Sumita Kachhwaha ${ }^{1,3^{*}}$ \\ ${ }^{1}$ Bioinformatics Infrastructure Facility (DBT-BIF), University of Rajasthan, Jaipur- 302004, Rajasthan, India \\ ${ }^{2}$ Amity Institute of Biotechnology, Amity University Rajasthan, Jaipur- 303002, Rajasthan, India \\ ${ }^{3}$ Departement of Botany, University of Rajasthan, Jaipur- 302004, Rajasthan, India
}

\section{*Corresponding author: Kachhwahasumita@rediffmail.com}

\begin{abstract}
Environmental stress constraints like temperature, drought and salinity affect plant growth, development and productivity negatively. In maize (Zea mays L.) water-deficiency affects flowering, pollination and embryo development. Transgenic plants having drought tolerance is a priority target in maize breeding programs worldwide. Various transcription factors play a key role in plant development and stress management. DRE-Binding Protein 2A (DREB2A) has been shown to play a central role in drought tolerance in Arabidopsis thaliana. The homologue of dreb2a in Zea mays is Zmdreb2a, a well-known transcription factor regulating genes induced under stress conditions. Studies indicated that Zmdreb2a transgenic plants show not only stress tolerance but also growth retardation. It is a major targeted gene for transgenesis to produce drought-tolerant plants. However, to make construct for transgenic plants, there is need to consider functional transcripts and regulating mechanism of Zmdreb2a mRNAs under drought condition. We have analyzed publicly available RNASeq data of leaf meristem, ovary and developing root and used a host of FastQC, Tophat, Cufflinks, Cuffmerge, Cuffdiff and ' $R$ ' packages to functionally characterize the differentially expressed genes (DEGs). In addition, prediction of protein structure, nuclear localization signals and transactivation domain were performed using cNLS mapper and 9aaTAD prediction tools. Alternative splicing of Zmdreb2a pre-mRNA was shown to be strongly associated with developmental stage and isoforms show fluctuating expression under stress. This study provides putative functional transcripts of Zmdreb2a, and NMD as a regulating mechanism to control their abundance.
\end{abstract}

Keywords: DREB2A, Transcription factors, Drought, Environmental stress, RNA sequencing, Splicing, NMD, R packages, Fastqc, Tophat.

Abbreviations: Zmdreb2a_Zea mays dehydration-responsive element binding protein 2A, Nonsense mediated decay, DEG_ Differentially expressed genes.

\section{Introduction}

Environmental stress constraints like temperature, drought and salinity affect plant growth, development and productivity negatively (Golldack et al., 2014). Extreme weather disaster have further aggravated the crop production across the globe as repeated in a study for the period 1964-2007 (Lesk et al., 2016). A recent study stated that maize production had declined to $39.3 \%$ with reduction in $40 \%$ water supply (Daryano et al., 2016). Maize (Zea mays L.) is among the highly consumed staple crops and its production is frequently impacted by water scarcity (Boyer et al., 2004). Water-deficiency adversely affects flowering, pollination and embryo development (Xu et al., 2014).

Plants sense environmental stimuli and transduce information to downstream components. This signaling in turn contributes to regulation and direct protection of plant cells through various type of physiological and biochemical interactions (Shinozaki et al., 2003; Nakashima et al., 2014). Since past two decades, several genes have been reported that are induced under such stressful conditions and their products are thought to function in stress tolerance response (Gong et al., 2014). These genes include key metabolic enzymes, late embryogenesis-abundant (LEA) proteins, detoxification enzymes, chaperones, protein kinases and transcription factors (Maruyama et al., 2009). Cis and trans-acting factors have been extensively studied in many plant species. They regulate stress-inducible genes either by ABA-dependent or ABA-independent mechanism (Yoshida et al., 2014). Numerous researchers stated the crucial role played by transcription factors and consensus sequences in drought conditions. The dehydrationresponsive element (DRE), a well-studied cis-acting promoter element primarily regulates gene expression in response to dehydration, high salinity and cold (Kim at al., 2011). Other examples of cis-acting elements include Crepeat (CRT) and low-temperature responsive element (LTRE). These elements possess an A/GCCGAC motif which constitutes the core of DRE sequence (Baker et al., 1994; Sakuma et al., 2006).

DRE-Binding Protein 2A (DREB2A) has been shown to play a central role in drought tolerance in Arabidopsis thaliana (Liu 
et al., 1998). It is a member of APETALA2/EthyleneResponsive Factor (AP2/ ERF) superfamily of transcription factors (Dey et al., 2015). It binds with DRE in the promoter region of downstream genes and functions as a transcriptional activator (Nakashima et al., 2000; Sakuma et al., 2002). Orthologues of dreb2a have been reported in Rice, Wheat, Barley, Maize, etc. (Matsukura et al., 2010; Egawa et al., 2006; Qin et al., 2007; Xue et al., 2004).

For the study of gene expression pattern, many approaches are employed, viz. tag based platforms like serial analysis of gene expression (SAGE), massively parallel signature sequencing (MPSS) and microarray (Lipshutz et al., 1999; Brenner et al., 2000; Ekman et al., 2003). Although these approaches assist in determining expression profiles of mRNA species in a cell, there are technical shortcomings associated with such platforms(Han et al., 2015). Tag based platforms are restricted by biases introduced during cloning steps and doesn't enable us to identify new genes and alternative splice isoforms. Microarray platform, on the other hand, is biased towards non-specific crosshybridization of CDNA and the probes differ in hybridization properties. Additionally it only detects expression level of genes of interest, thus does not fully sample the whole transcriptome (Liu et al., 2007; Imadi et al., 2015; Tan et al.). As a result of these limitations, sequence based expression profiling of genes have been developed which offers accurate investigation of expression level on a genome-wide scale (Wang et al., 2009). One of such techniques is the ultra-high-throughput sequencing of messenger RNA (mRNA), widely known as RNA sequencing (RNA-seq) or transcriptome sequencing technology (Han et al., 2015). It offers computing expression of mRNA as well as non-coding RNAs and relies on conversion of messenger RNA (mRNA) to complementary DNA (cDNA), which is then sequenced into short read segments of $36-150$ base pairs. Most of the protein-coding genes in plants have introns. After transcription introns are removed through the splicing of precursor mRNA transcripts (pre-mRNAs). Splicing is performed by large ribonucleoprotein (RNP) complex (Spliceosome), that recognise splice sites in pre-mRNA molecule and then removes the introns (Shang et al., 2017). RNASeq data provides functionality to find Pattern of splicing in sequenced reads.

The availability of complete genomic sequence of maize plant and improved data mining tools has greatly facilitated this investigation and comparison of DNA/RNA sequences. This could help in crop breeding and raising transgenic maize crops with improved productivity and stress tolerance. Although several genes have been reported by researchers which affect developmental stages of maize plant but deep insight into transcriptome still remains unrevealed. Researchers conducted few genomic studies on maize plant and had not thoroughly examined leaf meristem, ovary and root tissues. The primary objective of our study was to probe publicly available transcriptome datasets of these tissues and examine the expression pattern of $Z m d r e b 2 a$ transcripts under drought condition and development stage. With the recent release of improved version of maize reference genome and gene annotation (Assembly AGPV4; released December 2015), the present study became feasible.

\section{Results}

Principal Component Analysis between Vegetative and Reproductive Stage

To investigate whether samples display variability within replicates, we have performed Principal Component Analysis (PCA) for the study accession PRJNA172724 and PRJNA271595. The plot was generated by assigning values of principal component 1 (PC1) and principal component 2 (PC2) on $x$ - and $y$-axis respectively (Figure 1 ). For ovary (PRJNA172724) and developing root tissue (PRJNA271595), replicates were clustered together and separable according to conditions. Because RNA-sequencing is a quantitative assay, our candidate genes showing similar FPKM values across replicates (Table2). Hence we included all these datasets for further analysis.

\section{Alternative splicing}

Alternative splicing pattern was investigated for maize's leaf meristem, ovary and developing root tissue respectively. To improve the overall alignment and mapping accuracy, we had chosen bias- and multi-read correction in TopHat v.2.0 respectively (Trapnell et al., 2009). Using these parameters, greater than $90 \%$ alignment was achieved (Table 1). Zmdreb2a isoform variants were acknowledged if the transcripts represent at least $10 \%$ of the total transcripts abundance in Cufflinks assembly (Trapnell et al., 2010). This resulted in identification of five splicing variants for leaf meristem, four for ovary tissue and seven for each of the root developmental zones (Figure 2). Out of these seven variants found in root tissue, four were found to have same annotation reported earlier in Ensembl Plants database [http://plants.ensembl.org]. The remaining three transcripts were not reported earlier and are thus novel. The dreb2a isoform sequences were extracted using coordinates from separately merged transcript files generated by Cuffmerge program (Roberts et al., 2011).

\section{Analysis of dreb2a variants identified in leaf meristem, ovary and root tissue}

While Cufflinks program assembles transcripts, Cuffdiff (Trapnell et al., 2013) estimates the differential expression of assembled transcripts. The results of the two studies were visualized using CummerBund:R package (Goff et al., 2013). Gene and transcript expression as well as annotation were retrieved from CummerBund (Table 2). In the RNAsequencing datasets, a gene was considered transcribed if the FPKM 95\% confidence interval lower boundary was greater than zero (Sekhon et al., 2013), as defined by Cufflinks. All the identified transcripts were merged in single file and compared with the reference Maize genome annotation retrieved from https://plants.ensembl.org. Our analysis identified three novel transcripts for dreb2a gene which shows differential exon usage. We set a minimal FPKM threshold of $95 \%$ confidence interval to consider a transcript is expressed. Based on this parameter, we had discarded one novel transcript Zm00001d010048 D1, and used Zm00001d010048_D and Zm00001d010048_D2 for further studies (Figure 2). 
Table 1. Mapping Summary Statistics for Poly (A) RNA-sequencing datasets.

\begin{tabular}{|c|c|c|c|c|c|c|c|}
\hline Study Accession & Description & Condition & Run Accession & Total Reads & $\begin{array}{l}\text { Processed } \\
\text { Reads }\end{array}$ & Mapped Reads & \% Mapped \\
\hline \multirow[t]{8}{*}{ PRJNA172724 } & Ovary & Watered & SRR536834 & 39043693 & 37990717 & 35922917 & 94.6 \\
\hline & Ovary & Watered & SRR536835 & 36621929 & 32145486 & 30498604 & 94.9 \\
\hline & Ovary & Drought & SRR536836 & 39642000 & 37869763 & 36157029 & 95.5 \\
\hline & Ovary & Drought & SRR536837 & 39355314 & 36420661 & 34666806 & 95.2 \\
\hline & Leaf Meristem & Watered & SRR536838 & 37801189 & 37231743 & 35571705 & 95.5 \\
\hline & Leaf Meristem & Watered & SRR536839 & 30659992 & 29072397 & 27482432 & 94.5 \\
\hline & Leaf Meristem & Drought & SRR536840 & 37455511 & 36900109 & 34751283 & 94.2 \\
\hline & Leaf Meristem & Drought & SRR536841 & 36908864 & 36330104 & 34471593 & 94.9 \\
\hline \multirow[t]{9}{*}{ PRJNA271595 } & Root tissue & Meristematic & SRR1740452 & 28466090 & 28466090 & 27034825 & 95 \\
\hline & Root tissue & Meristematic & SRR1740453 & 31091783 & 31091783 & 29506320 & 94.9 \\
\hline & Root tissue & Meristematic & SRR1740454 & 31119077 & 31119077 & 29549689 & 95 \\
\hline & Root tissue & Elongation & SRR1740455 & 30487579 & 30487579 & 28561920 & 93.7 \\
\hline & Root tissue & Elongation & SRR1740456 & 30596493 & 30596493 & 28239675 & 92.3 \\
\hline & Root tissue & Elongation & SRR1740457 & 30536114 & 30536114 & 29016602 & 95 \\
\hline & Root tissue & Differentiation & SRR1740458 & 26602730 & 26602730 & 25541495 & 96 \\
\hline & Root tissue & Differentiation & SRR1740459 & 27659894 & 27659894 & 26484718 & 95.8 \\
\hline & Root tissue & Differentiation & SRR1740460 & 29334683 & 29334683 & 27924359 & 95.2 \\
\hline
\end{tabular}

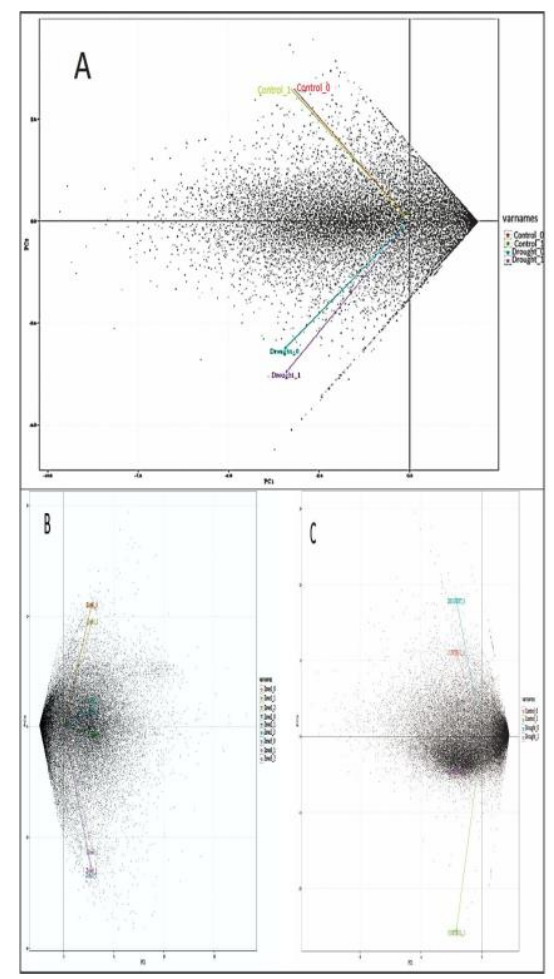

Fig 1. Principal Component Analysis showing similarity within replicates in (A) Ovary tissue (B) Developing root tissue (C) Leaf meristem. 
Table 2. Overall expression level of splicing variants (in FPKM value).

\begin{tabular}{|c|c|c|c|c|c|c|c|c|}
\hline \multirow[t]{2}{*}{ S. No. } & \multirow[t]{2}{*}{ Transcript Id } & \multicolumn{2}{|c|}{ Leaf Meristem } & \multicolumn{2}{|c|}{ Ovary Tissue } & \multicolumn{3}{|l|}{ Root Tissue } \\
\hline & & Control & Drought & Control & Drought & $\begin{array}{l}\text { Meristematic } \\
\text { Zone }\end{array}$ & $\begin{array}{l}\text { Elongation } \\
\text { Zone }\end{array}$ & $\begin{array}{l}\text { Differentiation } \\
\text { Zone }\end{array}$ \\
\hline 1. & Zm00001d010048_D & 3.57 & 1.6 & - & - & 3.0496 & 2.23993 & 4.38224 \\
\hline 2. & Zm00001d010048_D1 & - & - & - & - & 0.371623 & 0.40032 & 0.293744 \\
\hline 3. & Zm00001d010048_D2 & - & - & - & - & 0.945584 & 0.511662 & 0.61628 \\
\hline 4. & $\begin{array}{l}\text { Zm00001d010048_T0 } \\
01\end{array}$ & 2.93 & 0.57 & 2.51 & 0.72 & 8.82191 & 5.61216 & 6.50909 \\
\hline 5. & $\begin{array}{l}\text { Zm00001d010048_T0 } \\
02\end{array}$ & 0 & 0 & 0.05 & 0 & 0.771343 & 1.84107 & 9.07724 \\
\hline 6. & $\begin{array}{l}\text { Zm00001d010048_T0 } \\
03\end{array}$ & 12.29 & 14.27 & 9.27 & 13.23 & 12.585 & 7.02658 & 10.9858 \\
\hline 7. & $\begin{array}{l}\text { Zm00001d010048_T0 } \\
04\end{array}$ & 4.06 & 1.17 & 3.05 & 3.29 & 2.49606 & 0.917016 & 1.94569 \\
\hline \multicolumn{2}{|c|}{ Zmdreb2a } & 22.85 & 17.61 & 14.88 & 17.24 & 29.04 & 18.55 & 33.81 \\
\hline
\end{tabular}

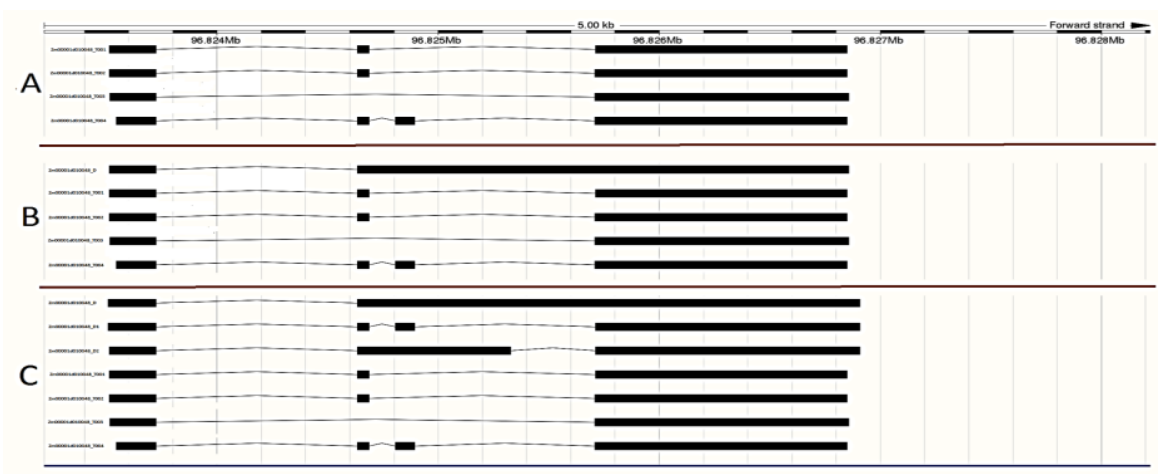

Fig 2. Splicing variants of Zmdreb2a identified in (A) Ovary Tissue (B) Leaf Meristem (C) Root tissue.

Table 3. Overview of transcripts identified through analyzing RNA-sequencing datasets .

\begin{tabular}{|c|c|c|c|c|c|c|}
\hline S. No. & Transcript Name & Locus & $\begin{array}{l}\text { DNA Binding } \\
\text { Domain } \\
\text { (ERF/AP2) }\end{array}$ & $\begin{array}{l}\text { Transcriptional } \\
\text { Activation Domain } \\
\text { (TAD) }\end{array}$ & $\begin{array}{l}\text { nuclear localization } \\
\text { signal (NLS) }\end{array}$ & Length (in amino acids) \\
\hline 1. & Zm00001d010048_D1 & $\begin{array}{l}\text { 8:96823507- } \\
96826907\end{array}$ & $\checkmark$ & $\checkmark$ & $\checkmark$ & 366 \\
\hline 2. & Zm00001d010048_T003 & $\begin{array}{l}\text { 8:96823514- } \\
96826858\end{array}$ & $\checkmark$ & $\checkmark$ & $\checkmark$ & 320 \\
\hline 3. & Zm00001d010048_T004 & $\begin{array}{l}8: 96823544- \\
96826850\end{array}$ & $\checkmark$ & $\checkmark$ & $\checkmark$ & 367 \\
\hline 4. & Zm00001d010048_D & $\begin{array}{l}\text { 8:96823507- } \\
96826907\end{array}$ & $x$ & $x$ & $x$ & 57 \\
\hline 5. & Zm00001d010048_D2 & $\begin{array}{l}\text { 8:96823511- } \\
96826907\end{array}$ & $x$ & $x$ & $x$ & 57 \\
\hline 6. & Zm00001d010048_T001 & $\begin{array}{l}\text { 8:96823511- } \\
96826849\end{array}$ & $x$ & $x$ & $x$ & 88 \\
\hline 7. & Zm00001d010048_T002 & $\begin{array}{l}\text { 8:96823512- } \\
96826849\end{array}$ & $x$ & $x$ & $x$ & 90 \\
\hline
\end{tabular}




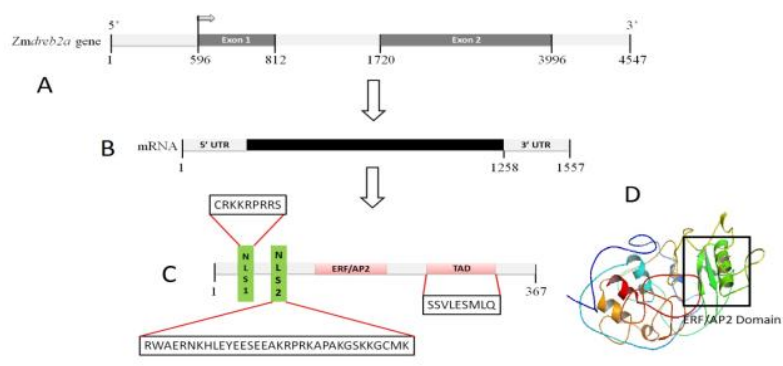

Fig 3. Domain architecture of Zmdreb2a showing structure of gene, mRNA and protein.

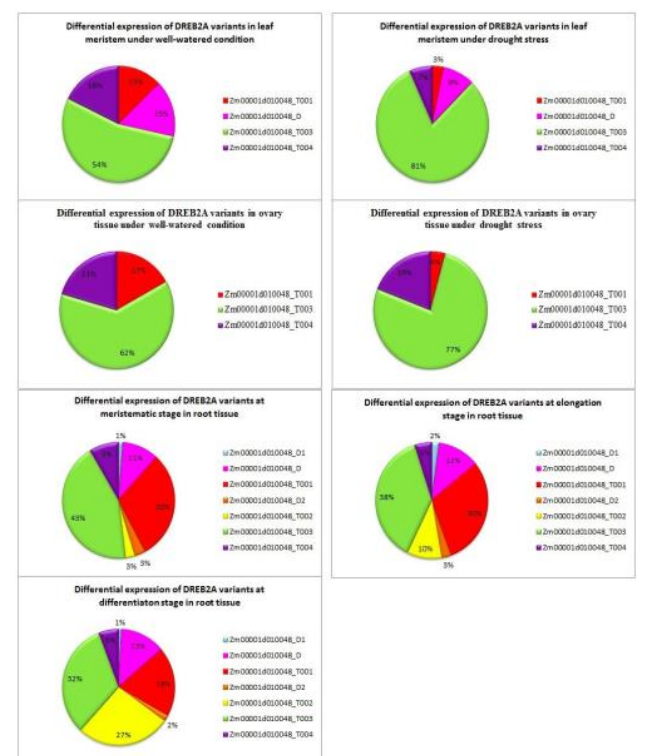

Fig 4. Differential Expression of Zmdreb2a splicing variants.

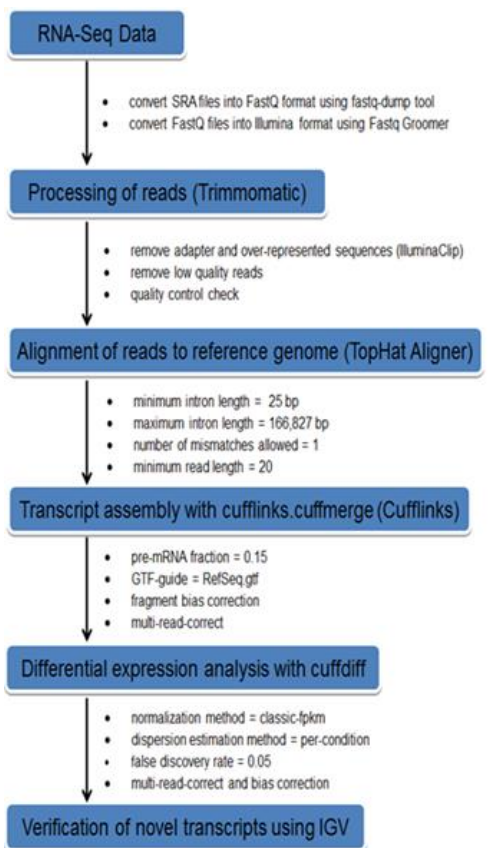

Fig 5. An overview of RNA-seq pipeline for identifying dreb2a variants in maize transcriptome. 


\section{Prediction of Zmdreb2a domains and structure}

The protein sequences of all the identified splicing variants were analyzed for domain architecture. We first explored and annotated the protein domains by using SMART- Simple Modular Architecture Research Tool (Schultz et al., 2013) and 9aaTAD Prediction Tool (Piskacek et al., 2007). The results confirmed the presence of two domains in DREB2A protein. These include an ERF/AP2 DNA binding domain (DBD) and transcriptional activation domain (TAD). We also searched for nuclear localization signal (NLS) using cNLS Mapper tool (Kosugi et al., 2009). cNLS mapper predicted two signals, viz. monopartite NLS1 and bipartite NLS2. We consider DREB2A protein to be functional only if it contains NLS signal and the identified domains. These domains and signal were present in three translated transcripts viz. Zm00001d010048_D1, Zm00001d010048_T003 and Zm00001d010048_T004 (Table 3). The gene, mRNA and protein structure were given in Figure 3.

\section{Annotation of expressed gene part using expressed sequence tags (ESTs) analysis}

ESTs are used to define expressed part of a gene within an organism's genome. The longest transcript Zm00001d010048_T004 was aligned against ESTs database (ESTdb) using NCBI MEGABLAST suite accessed through NCBI's BLAST services on the internet (NCBI). The alignment of transcript sequence over ESTs sequence confirmed the splicing pattern identified for Zmdreb2a gene with a threshold alignment E-value 6e-127. Each part of the transcript sequence matches to dreb2a gene found in other plant genus like Saccharum, Panicum, Sorghum, etc. (Supplementary File 1) which supported our RNA-seq pipeline and datasets.

\section{Differential Expression of Zmdreb2a}

We initially measured differential expression of dreb2a gene in order to understand how much gene is expressed in maize tissues under different conditions and developmental stage. Expression level of dreb2a was assessed by fragment per kilobase per million reads (FPKM) value using Cuffdiff and CummeRbund. An extremely low expression cutoff of 1.0 fragment per kilobase per million reads (FPKM) was applied and the transcripts were filtered accordingly. The expression level and transcript abundance of $d r e b 2 a$ isoform variants was estimated. The dehydration-responsive element binding protein $2 \mathrm{~A}($ dreb2a) expression was more in leaf meristem under well-watered conditions as compared to water scarcity. In contrast, it shows higher expression level in ovary tissue under drought stress (Table 2). The expression of dreb2a was also observed in developmental stages of root tissue. The splicing variants showed fluctuating expression in leaf meristem and ovary tissue under drought condition (Figure 4). The cDNA sequences for these transcripts are available in Supplemental File 1.

\section{Discussion}

Plants guard themselves against water deficit by relaying information in the form of external signals through a cascade of signaling events. These events alter the normal cellular activities, such as movement, secretion, enzyme activity, and gene expression. Many researchers studied dreb2a orthologue in Zea mays (Zmdreb2a). Under stress conditions, mainly drought, high salinity and cold, it induces expression of numerous genes in an ABA-independent manner (Gong et al., 2014; Maruyama et al., 2009; Yoshida et al., 2014). This enables plants to acquire immunity and help them to attain growth and development under hostile climatic conditions. The overall expression level of a gene, given by Cuffdiff, is calculated by summing expression of all its related transcripts. Although several studies have reported the pivotal role played by DREB proteins in acquiring resistance against drought stress, little is known about the expression of Zmdreb2a isoforms in different developmental stages and conditions. The present study is an attempt to answer this question.

To investigate the expression and pattern of isoforms, tuxedo protocol (Trapnell et al., 2013) of RNA-seq was employed. It uses TopHat for aligning spliced sequence reads. Isoform expression and alternative splicing pattern is the final outcome of our analysis. Alternative splicing is an important regulatory mechanism responsible for transcriptome and proteome diversity of higher eukaryotes. The splicing methods include exon skipping, intron retention, alternative donor and acceptor sites, and alternative position (Lorkovic et al., 2000). While exon skipping is common in animals, intron retention with transposable reads are frequently observed in plant genomes (lida et al., 2004; Wang et al., 2006; Filichkin et al., 2010). The present study revealed that differential usage of exon region is a major mechanism for alternative splicing in $Z m d r e b 2 a$. The pre-mRNA of $d r e b 2 a$ gene undergoes splicing events and generates variants which showed differential expression in developmental stage-specific manner and expression of isoforms fluctuate during drought stress. For example, Zm00001d010048_D is expressed in meristematic tissue of leaf and developing root tissue and thought to be development specific. Moreover RNA-sequencing data revealed that maize's dreb2a gene consists of two exons and one intron. The major exon is of 2275 nucleotides in length while the other exon is of 216 nucleotides.

We analyzed cDNA sequences of all the transcripts and found that not all transcripts make a full length protein. Instead there are only two functional transcripts which make a full length protein. Remaining transcripts have premature stop codon and are potential product of non-sense mediated decay. The functional transcripts contain an ERF/AP2 DNA binding domain, transcription activation domain and nuclear localization signal. Two functional transcripts coded full length protein contains AP2 domain, TAD and NLS. In addition, functional transcripts also have GCC box (GCCGAC box) a binding site for AP2 domain. So transcript abundance is thought to be regulated at pre-mRNA level. The present study proposes that translation of functional transcript is regulated by negative feedback mechanism using binding site for its own protein product (in nucleus at pre-mRNA stage).

It has also been reported by several scientists that when Zmdreb2a is used for making transgenic stress tolerant plants, these plants showed growth retardation (Liu et al., 1998; ). Expression of dreb2a not only increases during stress condition, but present results shows that it is also expressed in developmental zones of root tissues. Thus it 
may play a role in development of maize plant. Hence consecutive expression of Zmdreb2a in transgenic plants may affect developmental genes and retardation in growth was therefore, observed. Future studies are needed to manipulate the expression of Zmdreb2a to produce a transgenic plant with normal growth under drought condition.

To conclude, Zmdreb2a isoforms show variation in expression level according to climatic conditions and developmental stage and transcript abundance regulated by Non sense mediated decay.

\section{Materials and Methods}

\section{RNA-seq data}

Transcriptome of Maize cv. B73 samples are retrieved from NCBI as a SRA files. SRA files contain sequenced fragments of CDNA with attached adapter. SRA of vegetative and reproductive tissue were retrieved from study accession PRJNA172724

(https://www.ncbi.nlm.nih.gov/bioproject/PRJNA172724)

and SRA of root developmental zones were retrieved from PRJNA271595

(https://www.ncbi.nlm.nih.gov/bioproject/PRJNA271595). A summary of RNA-seq datasets is provided in Table 1 . These datasets contain single end reads of Poly (A) RNA sequences. SRA files were converted into fastq files using the fastqdump tool (version 2.5.7; http://www.ncbi.nlm.nih.gov/sra). The fastq files were then converted into Illumina format using FASTX-toolkit (http://hannonlab.cshl.edu/fastx_toolkit/index.html). As baseline reference and annotation, we used the latest Zea mays B73 genome assembly (RefGen_v4; released December 2015) downloaded from Ensembl Plant database (http://plants.ensembl.org).

\section{Processing of RNA-seq datasets}

In addition to machine error, high-throughput sequencing often generates short read sequences comprising primers and adapters. Such type of contamination may negatively affect the mappability and produce erroneous assembly. Hence, RNA sequence reads were processed using Trimmomatic toolkit v0.32.3. Adapter and Illumina-specific sequences, commonly referred to as overrepresented sequences were trimmed by running IlluminaClip program. Furthermore, crop, head crop, minimum length, sliding window, leading and trailing approaches were used to remove low quality bases requiring a minimum Phred score of 20. Finally, processed sequence files underwent quality control checks using FastQC tool (Andrews et al., 2010).

\section{Mapping of RNA-Seq reads using TopHat}

After processing and trimming, available single end reads were aligned to the reference Zea mays cv. B73 v.3.32 genome using TopHat v.2.0. In order to identify splice junctions, a minimum anchor length of 8 was fixed and only single mismatch were allowed. The minimum and maximum length of introns was set to 18 base pairs and 908 base pairs respectively.

\section{Transcript assembly using Cufflinks}

The read alignments from TopHat were assembled into a parsimonious set of transcripts using Cufflinks version 2.2.1.0 with parameters set at: " -0.05 Isoform fraction-GTFguide RefSeq.gtf -fragment bias correction -multi-readcorrect". Cufflinks uses reference annotation based transcript (RABT) assembly method (Roberts et al., 2011) to assemble against a known reference annotation. Transcript assembly allows for identification of splice variants, novel exons and novel full length transcripts. We obtained RefSeq gene annotation for all known genes in the Maize genome, as provided by the Ensembl Plant database. Cufflinks also measure transcript abundances in FPKM (Fragments Per Kilobase of transcript per Million fragments mapped), which is analogous to single-read RPKM (Reads Per Kilobase of transcript per Million reads mapped).

\section{Statistical analysis}

Principal component analysis (PCA) is performed for classification. PCA is an effective mathematical algorithm of reducing the dimensionality of datasets while retaining most of the variation in the data set. It identifies a new set of variables known as "principal components" to summarize the features of the data. The axes on the PCA plot are combinations of two new variables that are chosen to explain most of the variation in data.

\section{Comparison to reference annotation and differential analysis}

Once transcript assembly is completed, the merged transcript file from Cuffmerge was used as input to Cuffdiff along with reference sequence file AGPv3.32.dna.toplevel.fas downloaded from Ensembl Plants database. Cuffdiff version 2.2.1.3 was employed on the combined transcripts for identification of differentially expressed genes/transcripts. $\mathrm{R}$ package CummerBund was used for further analysis.

\section{Acknowledgments}

This work was financially supported by grants received in the form of BIF from Department of Biotechnology, Government of India. We are highly thankful to Professor S.C. Maheshwari for his valuable guidance.

\section{Supplementary Materials:}

Supplementary sequences 1: The complete sequences of identified transcripts.

Supplemental Figure 1: Validation of expressed Zmdreb2a gene part using expressed sequence tags.

\section{References}

Andrews S (2010) FastQC: a quality control tool for high throughput sequence data. Available online at:http://www.bioinformatics.babraham.ac.uk/projects/fa stqc. 
Baker SS, Wilhelm KS, Thomashow MF (1994) The 5'-region of Arabidopsis thaliana cor15a has cis-acting elements that confer cold-, drought- and ABA-regulated gene expression. Plant Mol. Biol. 24: 701-713.

Brenner S, Johnson M, Bridgham J, Golda G, Lloyd DH, Johnson D, Luo S, McCurdy S, Foy M, Ewan M, Roth R, George D, Eletr S, Albrecht G, Vermaas E, Williams SR, Moon K, Burcham T, Pallas M, DuBridge RB, Kirchner J, Fearon K, Mao Jl, Corcoran K (2000) Gene expression analysis by massively parallel signature sequencing (MPSS) on microbead arrays. Nat Biotechnol. 18: 630-634.

Boyer JS, Westgate ME (2004) Grain yields with limited water. J Exp Bot. 55: 2385-2394.

Daryanto S, Wang L, Jacinthe PA (2016) Global synthesis of drought effects on maize and wheat production. PLoS One 11: 1-15.

Dey S, Corina Vlot A (2015) Ethylene responsive factors in the orchestration of stress responses in monocotyledonous plants. Front Plant Sci. 6: 1-7.

Egawa C, Kobayashi F, Ishibashi M, Nakamura T, Nakamura C, Takumi S (2006) Differential regulation of transcript accumulation and alternative splicing of a DREB2 homolog under abiotic stress conditions in common wheat. Genes Genet Syst. 81: 77-91.

Ekman DR, Lorenz WW, Przybyla AE, Wolfe NL, Dean JFD, Warnell DB (2012) SAGE analysis of transcriptome responses in arabidopsis roots exposed to 2,4,6trinitrotoluene. Plant Physiol. 1: 2-4.

Filichkin SA, Priest HD, Givan SA, Shen R, Bryant DW, Fox SE, Wong W-K, Mockler TC (2010) Genome-wide mapping of alternative splicing in Arabidopsis thaliana. Genome Res. 20: 45-58.

Goff L, Trapnell C, Kelley D (2013) cummeRbund: Analysis, exploration, manipulation, and visualization of Cufflinks high-throughput sequencing data. R package version 2.8.2.

Golldack D, Li C, Mohan H, Probst N (2014) Tolerance to drought and salt stress in plants : unraveling the signaling networks. Front Plant Sci. 5: 1-10.

Gong F, Yang L, Tai F, Hu X, Wang W (2014) “'Omics"' of Maize Stress Response for Sustainable Food Production: Opportunities and Challenges. Omi A J Integr Biol. 18.

Han Y, Gao S, Muegge K, Zhang W, Zhou B (2015) Advanced applications of RNA sequencing and challenges. Bioinform. Biol. Insights 9: 29-46.

lida K, Seki M, Sakurai T, Satou M, Akiyama K, Toyoda T, Konagaya A, Shinozaki K (2004) Genome-wide analysis of alternative pre-mRNA splicing in Arabidopsis thaliana based on full-length cDNA sequences. Nucleic Acids Res. 32: 5096-5103.

Imadi SR, Kazi AG, Ahanger MA, Gucel S, Ahmad P (2015) Plant transcriptomics and responses to environmental stress: an overview. J Genet. 94: 525-537.

Kosugi S, Hasebe M, Tomita M, Yanagawa H (2009) Systematic identification of cell cycle-dependent yeast nucleocytoplasmic shuttling proteins by prediction of composite motifs. Proc Natl Acad Sci U S A 106: 1-6. doi:10.1073/pnas.0900604106.

Kim S, Mizoi J, Yoshida T, Fujita Y, Nakajima J, Ohori T, Todaka D, Nakashima K, Hirayama T, Shinozaki K, Yamaguchi-Shinozaki K (2011) An ABRE promoter sequence is involved in osmotic stress-responsive expression of the DREB2A gene, which encodes a transcription factor regulating drought-inducible genes in Arabidopsis. Plant Cell Physiol. 52: 2136-2146.

Lesk C, Rowhani P, Ramankutty N (2016) Influence of extreme weather disasters on global crop production. Nature 529: 84-87. Nature Publishing Group.

Lipshutz RJ, Fodor SPA, Gingeras TR, Lockhart DJ (1999) High density synthetic oligonucleotide arrays. Nat Genet. 21: 20-24.

Liu F, Jenssen T-K, Trimarchi J, Punzo C, Cepko C, OhnoMachado L, Hovig E, Patrick Kuo W (2007) Comparison of hybridization-based and sequencing-based gene expression technologies on biological replicates. BMC Genomics. 8: 153.

Liu Q, Miura S, Abe H, Shinozaki K, Yamaguchi-Shinozaki K, Sakuma Y, Kasuga M (2007) Two transcription factors, DREB1 and DREB2, with an EREBP/AP2 DNA binding domain separate two cellular signal transduction pathways in drought- and low-temperature-responsive gene expression, respectively, in Arabidopsis. Plant Cell 10: 1391.

Maruyama K, Takeda M, Kidokoro S, Yamada K, Sakuma Y, Urano K, Fujita M, Yoshiwara K, Matsukura S, Morishita Y, Sasaki R, Suzuki H, Saito K, Shibata D, Shinozaki K, Yamaguchi-Shinozaki K (2009) Metabolic pathways involved in cold acclimation identified by integrated analysis of metabolites and transcripts regulated by DREB1A and DREB2A. Plant Physiol. 150: 1972-1980.

Matsukura S, Mizoi J, Yoshida T, Todaka D, Ito Y, Maruyama K, Shinozaki K, Yamaguchi-Shinozaki K (2010) Comprehensive analysis of rice DREB2-type genes that encode transcription factors involved in the expression of abiotic stress-responsive genes. Mol Genet. Genomics 283: 185-196.

Nakashima K, Shinwari KZ, Sakuma Y, Motoski S, Setsuko M, Shinozaki K, Yamaguchi-Shinozaki K (2000) Organization and expression of two Arabidopsis DREB2 genes encoding DRE-binding proteins involved in dehydration- and highsalinity-responsive gene expression. Plant Mol. Biol. 42: 657-665.

Nakashima K, Yamaguchi-shinozaki K, Shinozaki K (2014) The transcriptional regulatory network in the drought response and its crosstalk in abiotic stress responses including drought, cold, and heat. Front Plant Sci. 5: 1-7.

Lorkovi ZJ, Wieczorek Kirk DA, Lambermon MH, F.W. 2000. Pre-mRNA splicing in higher plants. Trends Plant Sci. 5: 1360-1385.

Piskacek S, Gregor M, Nemethova M, Grabner M, Kovarik P, Piskacek M (2007) Nine-amino-acid transactivation domain: Establishment and prediction utilities. Genomics 89: 756-768.

Qin F, Kakimoto M, Sakuma Y, Maruyama K, Osakabe Y, Tran LSP, Shinozaki K, Yamaguchi-Shinozaki K (2007) Regulation and functional analysis of $Z m D R E B 2 A$ in response to drought and heat stresses in Zea mays L. Plant J. 50: 5469.

Roberts A, Pimentel H, Trapnell C, Pachter L (2011) Identification of novel transcripts in annotated genomes using RNA-seq. Bioinformatics 27: 2325-2329..

Sakuma Y, Maruyama K, Qin F, Osakabe Y, Shinozaki K, Yamaguchi-Shinozaki K (2006) Dual function of an Arabidopsis transcription factor DREB2A in water-stressresponsive and heat-stress-responsive gene expression. Proc Natl Acad Sci. USA. 103: 18822-18827. 
Schultz J, Milpetz F, Bork P, Ponting CP (1998) SMART, a simple modular architecture research tool: Identification of signaling domains. Proc Natl Acad Sci. USA. 95: 58575864.

Sakuma Y, Liu Q, Dubouzet JG, Abe H, Shinozaki K, Yamaguchi-Shinozaki K (2002) DNA-binding specificity of the ERF/AP2 domain of Arabidopsis DREBs, transcription factors involved in dehydration- and cold-inducible gene expression. Biochem. Biophys. Res. Commun. 290: 9981009.

Shinozaki K, Yamaguchi-shinozaki K, Seki M (2003) Regulatory network of gene expression in the drought and cold stress responses. Curr Opin Plant Biol. 6: 13-15.

Shang X, Cao Y, Ma L (2017) Alternative splicing in plant genes: A means of regulating the environmental fitness of plants. Int J Mol Sci. 18.

Schnable PS, Ware D, Cordes M, Carpita NC, Estill JC, Mead K, Yan L., Kim W, Soderlund C, Talag J, Rock SM, Springer NM, Vaughn MW, Shah N, Higginbotham J, Campos D, Li P, Jiang $N$, Zhu $Q$, Waligorski J, Chen W, Scara G, Graves TA, Yu Y, Ren L, Stein JC, Ambroise C, Kalyanaraman A, Henke J, Ko A, Minx P, Nascimento L, Spiegel L, Ponnala L, Levy MJ, Nagel DH, Chinwalla A, Fu Y, Rajasekar S, Abbott RM, Wei F, Nettleton D, Pasternak S, Chia J-M, Liang C, Kramer $M$, Lin J, Myers AM, Ruppert J, Penning BW, Lee $H$, Miller B, Dujmic Z, Wei S, Kim K, Westerman R, Du F, Delgado B, Angelova $A$, Deragon J-M, Zuccolo A, Wing RA, Jiang J, Jackson SM, Presting GG, Zhou S, Sun $Q$, Applebaum E, Belter E, Lomeli R, Jeddeloh JA, Kim H, Muller S, Spooner W, Delehaunty K, McMahan L, Scimone A, Martienssen RA, Lee S, Hsia A-P, Wolfgruber TK, He R, Wessler SR, Kumari S, Rotter K, Lopez G, Wang T, Reily AD, Crouse K, Liu Z, Waterman $M$, Sgro J, Thane N, Ying K, Courtney L, Van Buren P, Kohlberg S, Dawe RK, Sharma A, Fronick C, Jia Y, Liu S, Falcone J, Yeh C-T, Hodges J, Ashley E, Baucom RS, Fulton RS, Marchetto $P$, Brutnell TP, Golser W, Wang $H$, McCann MC, Fan C, Chaparro C, Phelps L, Mueller T, Emrich SJ, Leonard S, McCombie WR, Lisch DR, Cotton M, Narechania A, Bennetzen JL, SanMiguel P, Kruchowski, SS, Wissotski M, Schwartz DC, Kanchi K, Courtney B, Fulton L, Schneider KL, Sebastian A, Zutavern T, Zhang L, Ingenthron E, Gillam B, Faga B, Tomlinson C, Collura K, Currie J, Han Y,
Levy A, Ochoa K, Barbazuk WB, Zhang J, Delaney K, Aluru, S, Braidotti M, Cardenas M, Kudrna D, Nguyen J, Yang L, Strong C, Clifton SW, Thane T, Wilson RK (2009) The B73 maize genome: complexity, diversity, and dynamics. Science. 326: 1112-1115.

Tan KC, Ipcho SVS, Trengove RD, Oliver RP, Solomon PS (2009) Assessing the impact of transcriptomics, proteomics and metabolomics on fungal phytopathology. Mol Plant Pathol. 10: 703-715.

Trapnell C, Hendrickson DG, Sauvageau M, Goff L, Rinn JL, Pachter $L$ (2012) Differential analysis of gene regulation at transcript resolution with RNA-seq. Nat Biotechnol. 31 46-53. Nature Publishing Group.

Trapnell C, Pachter L, Salzberg SL (2009) TopHat: Discovering splice junctions with RNA-Seq. Bioinformatics. 25: 11051111.

Trapnell C, Pachter L, Salzberg SL, Williams B, Pertea G, Mortazavi A, Kwan G, van Baren MJ, Salzberg SL, Wold BJ, Pachter L (2010) Transcript assembly and abundance estimation from RNA-Seq reveals thousands of new transcripts and switching among isoforms. Nat Biotechnol. 28: 511-515.

Wang B-B, Brendel V (2006) Genomewide comparative analysis of alternative splicing in plants. Proc. Natl. Acad. Sci. 103: 7175-7180.

Wang X, Elling AA, Li X, Li N, Peng Z, He G, Sun H, Qi Y, Liu XS, Deng XW (2009) Genome-wide and organ-specific landscapes of epigenetic modifications and their relationships to mRNA and small RNA transcriptomes in maize. Plant Cell 21: 1053-1069.

Xu J, Yuan Y, Xu Y, Zhang G, Guo X, Wu F, Wang Q, Rong T, Pan G, Cao M, Tang Q, Gao S, Liu Y, Wang J, Lan H, Lu Y (2014) Identification of candidate genes for drought tolerance by whole-genome resequencing in maize. BMC Plant Biol. 14: 1-15. BMC Plant Biology.

Xue GP, Loveridge CW (2004) HvDRF1 is involved in abscisic acid-mediated gene regulation in barley and produces two forms of AP2 transcriptional activators, interacting preferably with a CT-rich element. Plant J. 37: 326-339.

Yoshida T, Mogami J, Yamaguchi-Shinozaki K (2014) ABAdependent and $A B A$-independent signaling in response to osmotic stress in plants. Curr Opin Plant Biol. 21: 133-139. 